\title{
Digital Back-Propagation Performance in Wideband Optical Fibre Transmission Systems
}

\author{
Lidia Galdino(1), Gabriel Saavedra ${ }^{(1)}$, Daniel Semrau(1), Daniel J. Elson ${ }^{(1)}$, Domaniç Lavery ${ }^{(1)}$, \\ Mingming Tan ${ }^{(2)}$, Md Asif Iqbal ${ }^{(2)}$, Paul Harper ${ }^{(2)}$, Robert I. Killey ${ }^{(1)}$, Polina Bayvel(1)
}

(1) Optical Networks Group, Department of Electronic and Electrical Engineering, UCL, Torrington Place, London WC1E 7JE, UK. (I.galdino@ucl.ac.uk)

(2) Aston Institute of Photonic Technologies, Aston University, Birmingham B4 7ET, UK.

\begin{abstract}
Single channel digital back-propagation (SC-DBP) performance with different transmission bandwidths is experimentally and theoretically investigated. The SC-DBP gain reduces with transmission bandwidth; from $1.2 \mathrm{~b} / \mathrm{sym}$ for single channel to $0.2 \mathrm{~b} / \mathrm{sym}$ for C-band transmission at $2000 \mathrm{~km}$.
\end{abstract}

\section{Introduction}

In transmission over a conventional optical fibre link, which is fundamentally nonlinear, the achievable SNR is limited by nonlinear distortion. Several digital techniques are available in the literature to mitigate fibre nonlinearity, among them - digital back-propagation (DBP) ${ }^{1}$. Due to technological limitations of digital-to-analogue converters (DAC) and analogue-to-digital converters (ADC), practical implementation of wideband transmitter-side or receiver-side DBP using a single transceiver is currently challenging. For a single receiver, the highest bandwidth backpropagated that has been experimentally demonstrated is $120 \mathrm{GHz}^{2}$. Several reports on experimentally achievable gains for full-field DBP showed a doubling ${ }^{2}$ and tripling ${ }^{3}$ of transmission distances. Additionally, the achievable gains of single channel DBP (SC-DBP) on fully loaded $\mathrm{C}$ - and L-band transmission systems have been studied, showing approximately $0.5 \mathrm{~dB} Q^{2}$-factor improvement $^{4}$. The limits of SC-DBP as the transmission bandwidth increases have been only theoretically studied ${ }^{5}$

In this work, we experimentally investigated the performance of SC-DBP over different propagated bandwidths. A theoretical performance analysis of the experiental transmission system, for both linear electronic chromatic dispersion compensation (EDC) only and DBP nonlinear compensation, has also been carried out, providing a deeper theoretical understanding of the experimental results. The $\mathrm{GN}$-model ${ }^{9}$ and generalized expressions ${ }^{2}$ to estimate the signal-tonoise ratio (SNR) in the presence of transceiver noise are used to estimate the DBP performance. The mutual information (MI) is used as the metric to quantify the DBP benefits on achievable information rates. The channel under test is 40 GBd Nyquist-shaped dual polarization 64-ary quadrature amplitude modulation (DP-64QAM), analyzed for propagated bandwidths between $40 \mathrm{GHz}$ and 4.1 THz , and over transmission distances of up to $2000 \mathrm{~km}$.

\section{Transmission system}

The experimental setup used for transmission is shown in Fig. 1. Seven external cavity lasers (ECLs) with $100 \mathrm{kHz}$ linewidth and $41 \mathrm{GHz}$ frequency spacing were used as sources for four odd- and three even-indexed subcarriers. Each set of sources was modulated using an IQ modulator, with drive signals generated with root raised cosine (RRC) filters with $0.1 \%$ roll-off factor followed by $92 \mathrm{GS} / \mathrm{s}$ DACs, to obtain spectrallyshaped 40 GBd 64-QAM signals. The odd and even channels were decorrelated with a $15 \mathrm{~ns}$ delay and combined before being polarisationmultiplexed (PolMux) to form a $7 \times 40$ GBd DP64QAM superchannel. The superchannel signal was subsequently combined with spectrallyshaped amplified spontaneous emission (SSASE) noise, used to emulate additional interfering channels ${ }^{6,7}$, allowing a range of transmission bandwidths to be investigated. The inset in Fig. 1 illustrates the 7 subcarries plus the SS-ASE, covering 4.1 THz of transmission bandwidth.

For transmission, a recirculating fibre loop was used comprising a polarization scrambler (PS), a single span of $101 \mathrm{~km}$ Corning ${ }^{\circledR}$ Vascade ${ }^{\circledR}$ EX2000 with a total loss of $16.2 \mathrm{~dB}$, an EDFA, a wavelength selective switch (WSS) for adjustable gain flattening, and a second EDFA to overcome the loss of loop components.

At the receiver the optical signal was amplified and band-pass filtered at $1550 \mathrm{~nm}$. A separate ECL was used as the local oscillator, and de- 


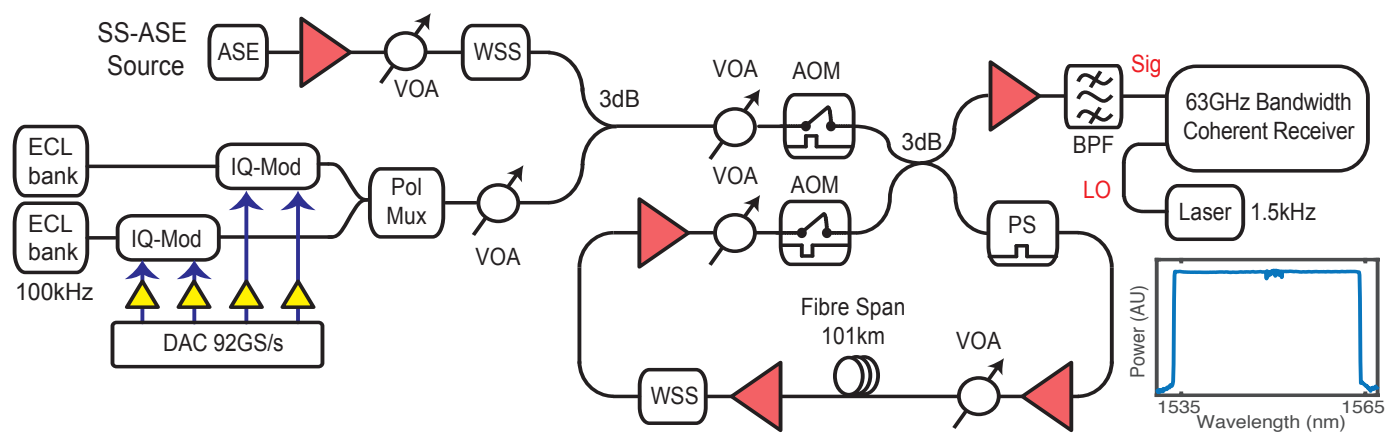

Fig. 1: Experimental configuration.

tection was carried out in a digital coherent receiver followed by digital signal processing (DSP) as $\mathrm{in}^{2,8}$. SNR and mutual information (MI) were used as the performance metrics for this system.

The SNR was evaluated as the ratio between the variance of the transmitted symbols $\mathbb{E}\left[|X|^{2}\right]$ and the variance of the noise $\sigma^{2}$, where $\sigma^{2}=$ $\mathbb{E}\left[|X-Y|^{2}\right]$ and $Y$ represents the received symbols. The MI was estimated from the received data via Monte Carlo integration and provides an upper bound on the performance for any coded system based on DP-64QAM ${ }^{8}$. In the experimental testbed used, the maximum SNR of $21 \mathrm{~dB}$ with a $\mathrm{Ml}$ of $11.8 \mathrm{~b} / \mathrm{sym}$ for single channel and 11.3 b/sym at an SNR of $19 \mathrm{~dB}$ for WDM.

In the theoretical analysis, a generalized formula to estimate the SNR for EDC and DBP that accounts for the transceiver noise and, in particular, the interactions between the signal and the co-propagating transceiver noise was used as described in ${ }^{2}$. Therefore, a transceiver limited SNR of $21 \mathrm{~dB}$ and $19 \mathrm{~dB}$ measured in the experimental testbed was considered in the analytical model for. The NLI coefficient was calculated using the GN model as detailed in ${ }^{9}$. The ASE noise power generated by the EDFA that compensates for the re-circulating loop loss components was also taken in to account on the analytical model.

\section{Results}

\section{Single channel transmission}

The DBP performance after transmission of a single 40 GBd DP-64QAM channel was first assessed to identify the upper bounds of the achievable DBP gains in the system under test. The performance improvements obtained by carrying out full-field DBP were then compared with the performance of single channel DBP over different transmitted bandwidths, of up to $4 \mathrm{THz}$.

Figure 2 illustrates the MI (at optimum signal launch power) as a function of distance for single channel transmission. The experimental measurements recorded that the $\mathrm{Ml}$ after transmission

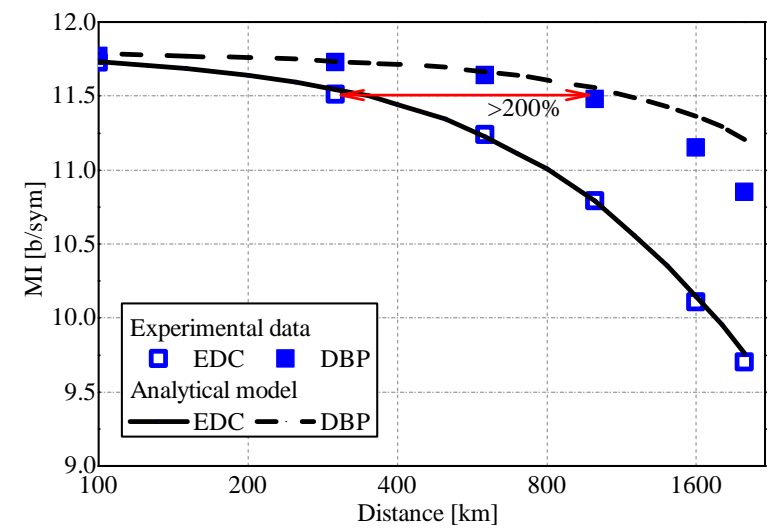

Fig. 2: Single channel transmission: MI vs. distance.

over $100 \mathrm{~km}$ using EDC only was $11.7 \mathrm{~b} / \mathrm{sym}$, reduced to $10.8 \mathrm{~b} / \mathrm{sym}$ at $1000 \mathrm{~km}$ and further reduced to $9.7 \mathrm{~b} / \mathrm{sym}$ at $2000 \mathrm{~km}$. When DBP was applied, the MI gain over the values obtained with EDC only increased with distance. For a fixed $\mathrm{MI}$ of $11.5 \mathrm{~b} / \mathrm{sym}$, EDC only allowed a transmission distance of $300 \mathrm{~km}$, while DBP achieved a reach of $1000 \mathrm{~km}$, a more than tripling of the transmission distance. Additionally, a gain in $\mathrm{MI}$ of $1.2 \mathrm{~b} / \mathrm{sym}$ by applying DBP was experimentally demonstrated at a transmission distance of $2000 \mathrm{~km}$. These are the highest gains in distance and/or MI that have been reported, to the best of our knowledge. A good agreement between the theoretical model and experimental data was found; at longer distances, the small discrepancy between the theoretical model and experimental results may be due to stochastic effects, such as polarization mode dispersion as described in ${ }^{2}$.

It is important to note that the Ml gain increases with the transmission distance; at a distance of $100 \mathrm{~km}$ the gain was $0.05 \mathrm{~b} / \mathrm{sym}$, increasing to $0.8 \mathrm{~b} / \mathrm{sym}$ at $1000 \mathrm{~km}$ and $1.2 \mathrm{~b} / \mathrm{sym}$ at $2000 \mathrm{~km}$. As illustrated in Fig. 2, at short transmission distances, the transceiver limited SNR is the predominant source of noise in the system. Thus, systems with finite SNR generally yield limited DBP performance over short transmission distances, where the link SNR is relatively high com- 


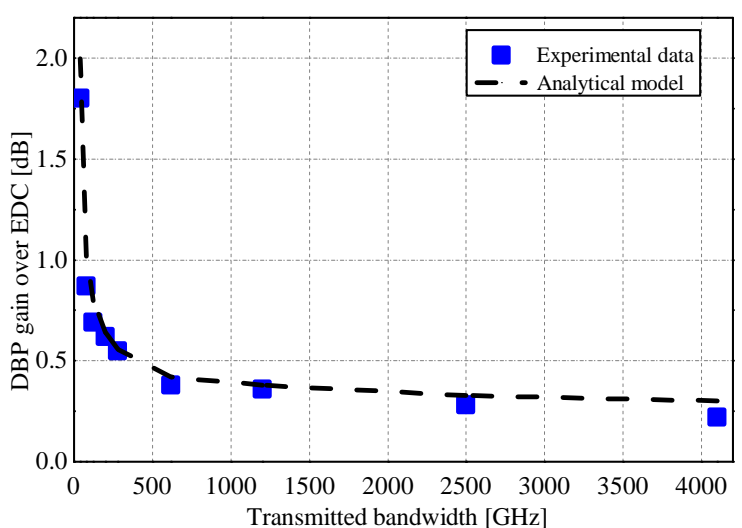

Fig. 3: SC-DBP gain over EDC at $1010 \mathrm{~km}$ for different transmitted bandwidths.

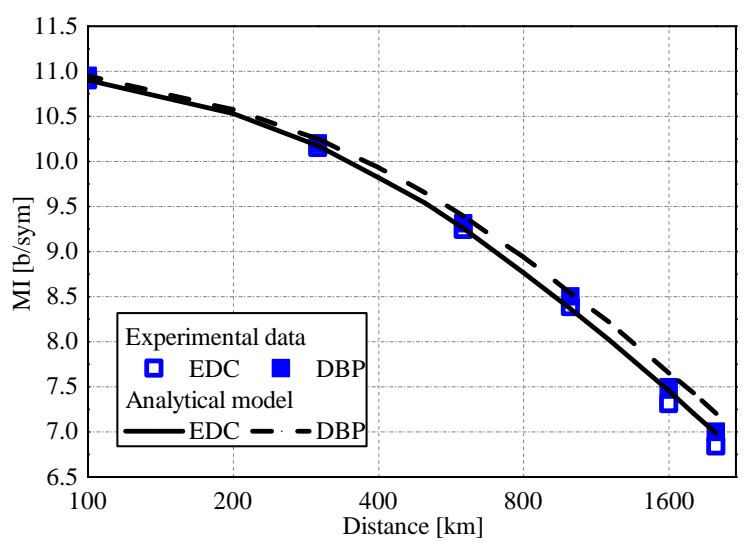

Fig. 4: 4.1 THz transmission: Ml vs. distance.

pared to transceiver noise sources. For longer distances, the system performance is dominated by accumulation of ASE and by nonlinear effects, which are compensated by applying DBP.

\section{Multi-channel transmission system}

The next step was to investigate the peformance of SC-DBP over different transmission bandwidths. As shown in Fig. 3, the SC-DBP gain over EDC was quantified for the propagated bandwidths of $40,80,120,200,280,620,1200,2500$ and $4100 \mathrm{GHz}$, after transmission over $1010 \mathrm{~km}$. A decrease in the gain offered by SC-DBP as the propagated bandwidth was increased can be observed. This reduction in SNR gain is due to the accumulation of NLI according to a logarithmic law for increasing bandwidth, as described in $^{7}$. The experimental results show a SNR gain of $1.8 \mathrm{~dB}$ for single channel transmission being reduced to $0.7 \mathrm{~dB}$ for a $120 \mathrm{GHz}$ propagated bandwidth, and further reduced to 0.4 and $0.25 \mathrm{~dB}$ for propagated bandwidths of 1.2 and $4.1 \mathrm{THz}$, respectively. These results are in line with the theoretical study from ${ }^{5}$.

To analyze the performance of SC-DBP in a fully loaded C-band system, Fig. 4 illustrates the $\mathrm{Ml}$ (at optimum launch power) as a function of distance for a $4.1 \mathrm{THz}$ propagated bandwidth. A marginal gain from SC-DBP over EDC can be observed; only $0.2 \mathrm{~b} / \mathrm{sym}$ gain after a $2000 \mathrm{~km}$ transmission distance. Despite this marginal gain, the $\mathrm{MI}$ gain relative to EDC only is also increased for longer transmission distances; from $0.03 \mathrm{~b} / \mathrm{sym}$ over $100 \mathrm{~km}$ to $0.2 \mathrm{~b} / \mathrm{sym}$ over $2000 \mathrm{~km}$.

\section{Conclusion}

SC-DBP performance was experimentally and theoretically studied for long haul transmission systems with signal propagated bandwidth to up to $4.1 \mathrm{THz}$. It was observed that the SC-DBP gain monotonically decreased with increasing propagated bandwidth. Although this effect was previously predicted theoretically, this is the first explicit and detailed experimental assessment of this, and it is in line with the GN-model predictions.

\section{Acknowledgment}

This work was supported by UK EPSRC Program Grant UNLOC EP/J017582/1, FP7 ITN programme ICONE (608099) and the RAEng under the Research Fellowships scheme.

\section{References}

[1] E. Ip, "Nonlinear compensation using backpropagation for polarization-multiplexed transmission," J. Lightw. Technol. 28(6), 939-951 (2010).

[2] L. Galdino, et al., "On the limits of digital back-propagation in the presence of transceiver noise," Optics Express 25(3), 4564-4578 (2010).

[3] E. Temprana, et al., "Demonstration of coherent transmission reach tripling byfrequency-referenced nonlinearity pre-compensation in EDFA-only SMF link," Proc. ECOC, (2016).

[4] A. Ghazisaeidi et al., "Submarine Transmission Systems Using Digital Nonlinear Compensation and Adaptive Rate Forward Error Correction," in J. of Lightw. Technol., 34(8) 1886-1895 (2016).

[5] R. Dar et al.,"On the limits of digital back-propagation in fully loaded WDM systems," Photn. Technol. Let. 28(11), 1253-1256 (2016).

[6] D. J. Elson, et al.."High spectral density transmission emulation using amplified spontaneous emission noise," Opt. Lett. 41(1), 68-71 (2016).

[7] G. Saavedra, et al.,"Experimental Investigation of Nonlinear Signal Distortions in Ultra-Wideband Transmission Systems," Proc. OFC, (2017).

[8] R. Maher, et al., "Increasing the information rates of optical communications via coded modulation: a study of transceiver performance," Sci. Rep. 6, 21278 (2016).

[9] P. Poggiolini, et al., "The GN-model of fiber non-linear propagation and its applications," J. Lightw. Technol. 32(4), 694-721 (2014). 